\title{
On the Massive Sine-Gordon Equation in the Higher Regions of Collapse
}

\author{
Francesco Nicolò
}

Istituto di Matematica dell'Università di Roma 1, I-00100 Roma, Italy and Istituto Nazionale di Fisica Nucleare - Sezione di Roma 1, I-00100 Roma, Italy

\begin{abstract}
We prove that the renormalization program discussed in [1] can also be developed beyond the $\bar{\alpha}^{2}=2 \pi(\sqrt{17}-1)$ threshold found in the preceding work. This result, as a byproduct, also allows a simplification in the technical part of the proof of ultraviolet stability in the $\varphi_{3}^{4}$-theory [2]. In the last section of this work we discuss, heuristically, but in some detail the interpretation of the sine-Gordon theory as a two-dimensional Yukawa gas for $\beta e^{2}=\alpha^{2}>4 \pi$.
\end{abstract}

\section{Introduction}

The two-dimensional sine-Gordon theory has been studied by Fröhlich [3] for the values of $\alpha^{2}<4 \pi$ and, in the finite volume, by Benfatto et al. [1] for the values $\geqq 4 \pi$. There it was proven that, for $\alpha^{2} \geqq 4 \pi$, the theory has to be renormalized; a renormalization procedure was constructed which amounted to subtracting from the potential $V_{0, I}^{(N)}=2 \lambda \int_{I}: \cos \alpha \varphi_{\xi}^{(N)}: d \xi$ some constant counterterms $C_{i}(N)$, whose number increases each time $\alpha^{2}$ overcomes the thresholds $\alpha_{2 n}^{2}=8 \pi(1-1 / 2 n)$ and which, of course, become infinite as the cutoff is removed $(N \rightarrow \infty)$. Although the procedure envisaged in [1] seemed to prove the ultraviolet stability of the theory for any values $\alpha^{2}<8 \pi$, some technical difficulties did not allow us to prove the upper bound of the ultraviolet stability for $\alpha^{2} \geqq \bar{\alpha}^{2}=2 \pi(\sqrt{17}-1)$.

The main goal of this paper is to prove that this spurious threshold can be removed. This is obtained by proving a theorem which allows us not to use the second part of Lemma 2 in [1], which was true only for $\alpha^{2}<\bar{\alpha}^{2}$. As this lemma was also used in the proof of the upper bound in the $\varphi_{3}^{4}$-theory [2] and as this result can be immediately translated for that field theory model, this amounts to a slightly technical simplification of that proof also. Moreover now the proofs of the upper and lower bound appear more symmetric. The theorem is applied to prove explicitly the ultraviolet stability for all the values of $\alpha^{2}<\frac{32}{5} \pi\left(\frac{32}{5} \pi>\bar{\alpha}^{2}\right)$, but the structure of this result and of the renormalization technique discussed in [1], allows us to conclude that the proof of stability for all $\alpha^{2}<8 \pi$ is only a matter of 
computation and no new ideas are necessary at all. After a short review of the renormalization scheme, discussed in Sect. 1, the next three sections are devoted to a careful discussion of this result. The last section is of a different and less technical nature. It is well known that the sine-Gordon theory has a very interesting interpretation, for $\alpha^{2}<4 \pi$, at short distances, as a classical two-dimensional Coulomb gas ${ }^{1}$ for enough high temperature $\left(\alpha^{2}=\beta e^{2}\right.$, where $\pm e$ is the particle charge) and as the counterterms in the renormalization procedure are constants we expect it must be possible to preserve the Coulomb gas interpretation also at lower temperatures, that is for $\alpha^{2} \geqq 4 \pi$. Therefore one is immediately challenged to understand which phenomena are produced in the Coulomb gas each time $\beta$ overcomes a $\frac{1}{e^{2}} \alpha_{2 n}^{2}$ threshold. This was briefly discussed in [1], where heuristic arguments were given to show that at each even threshold the gas tend to form neutral clusters of particles, whose size tends to zero as $N$ goes to infinity and whose density tends to infinity in the same limit. The renormalization procedure can be seen as a way of subtracting this infinite sea of clusters in such a way that the meaningful statistical observables refer to the gas of single particles which do not cluster and lie over this sea. This interpretation is discussed at some length in the last section where heuristic arguments are given to get the expression of the charge-density in the different regions of $\alpha^{2}$.

Some similar results for the massive Thirring model in the repulsive case have been obtained by Korepin [4], with different techniques (see also the references therein for the results on the sine-Gordon massless model using the quantum inverse scattering method).

\section{The Renormalization Scheme}

To prove the ultraviolet stability for a field theory, in a finite volume $I$, with an interaction potential $V_{I}\left(\varphi^{(N)}\right) \equiv V_{I}^{(N)}$ amounts to proving the following inequalities

$$
\exp \left[-E_{-}(\lambda)|I|\right] \leqq \int P\left(d \varphi^{(N)}\right) \exp V_{I}^{(N)} \leqq \exp \left[E_{+}(\lambda)|I|\right],
$$

where $\varphi^{(N)}$ is the gaussian field, depending on the cutoff $N$, with covariance

$$
C^{(N)}=(1-\Delta)^{-1}-\left(\gamma^{2(n+1)}-\Delta\right)^{-1},
$$

where $\Delta$ is the Laplace operator in $R^{2}$ and $\gamma>1$ is a constant (see [1]). Here $P\left(d \varphi^{(N)}\right)$ is the free field measure, $V_{I}^{(N)}$ is the renormalized potential and $E_{ \pm}(\lambda)$ are finite constants, $N$-independent.

In [1] we proved that, given the potential of the sine-Gordon theory in two dimensions

$$
V_{0, I}^{(N)}=2 \lambda \int_{I}: \cos \alpha \varphi_{\xi}^{(N)}: d \xi
$$

1 To be precise the massive sine-Gordon theory describes a Yukawa gas instead of the Coulomb one, but at short distances the properties of the two gases coincide, so from now on we will always refer to the "Coulomb gas" interpretation of this field theory model 
the renormalized potential for which (1.1) is fulfilled is for $\alpha^{2}$ in the interval $\left[4 \pi, \bar{\alpha}^{2}\right.$ )

$$
\bar{V}_{I}^{(N)}=V_{I}^{(N)}-\mathscr{E}_{N}\left(V_{0, I}^{(N)}\right)-\frac{1}{3 !} \mathscr{E}_{N}^{T}\left(V_{0, I ; 3}^{(N)}\right),
$$

where

$$
\begin{aligned}
V_{I}^{(N)} & =V_{0, I}^{(N)}-\sum_{1}^{2}{ }_{k} C_{2 k}(N) \\
& =V_{0, I}^{(N)}-\sum_{1}^{2} \frac{1}{(2 k) !} \mathscr{E}_{N}^{T}\left(V_{0, I}^{(N)} ; 2 k\right),{ }^{2}
\end{aligned}
$$

where $\mathscr{E}_{N}^{T}(f ; k)$ is the $k$-order cumulant performed with respect to the free field measure $P\left(d \varphi^{(N)}\right)$. For $\alpha^{2}<\alpha_{4}^{2}=8 \pi\left(1-\frac{1}{4}\right)=6 \pi$ only the cumulants until the second order have to be subtracted. More generally it was expected in [1] that each time $\alpha^{2}$ overcomes a threshold value $\alpha_{2 n}^{2}=8 \pi\left(1-\frac{1}{2 n}\right)$ a constant

$$
C_{2 n}(N)=\frac{1}{2 n !} \mathscr{E}_{N}^{T}\left(V_{0, I}^{(N)} ; 2 n\right)
$$

has to be subtracted, the renormalized potential looking, therefore:

$$
\begin{gathered}
V_{I}^{(N)}=V_{0, I}^{(N)}-\sum_{1}^{n} \frac{1}{(2 k) !} \mathscr{E}_{N}^{T}\left(V_{0, I}^{(N)} ; 2 k\right) \\
\alpha^{2} \in\left[\alpha_{2 n}^{2}, \alpha_{2(n+1)}^{2}\right) .
\end{gathered}
$$

This was proved in [1] for the lower bound, but for the upper bound the proof was lacking when $\alpha^{2} \geqq \bar{\alpha}^{2}$ and the elimination of this restriction is the main goal of this paper.

To prove (1.1), as discussed in detail in $[1,2], \varphi^{(N)}$ is decomposed as a sum of independent gaussian fields

$$
\varphi_{\xi}^{(N)}=\sum_{0}^{N} \tilde{\varphi}_{\xi}^{(k)}
$$

with covariance

$$
\tilde{C}_{k}(\xi-\eta)=\frac{1}{(2 \pi)^{2}} \int d p e^{i p(\xi-\eta)}\left(\frac{1}{\gamma^{2 k}+p^{2}}-\frac{1}{\gamma^{2(k+1)}+p^{2}}\right),
$$

then one performs the integration in (1.1) by successive integrations over the fields $\tilde{\varphi}^{(k)}$

$$
\int P\left(d \varphi^{(N)}\right) \exp V_{I}^{(N)}=\int P\left(d \varphi^{(N-1)}\right)\left\{\int P\left(d \tilde{\varphi}^{(N)}\right) \exp V_{I}^{(N)}\right\},
$$

and shows that

$$
\int P\left(d \tilde{\varphi}^{(N)}\right) \exp V_{I}^{(N)}=\exp \left[\tilde{V}_{I}^{(N-1)}+R^{(N-1)}(\hat{i})|I|\right]
$$

where $\tilde{V}_{I}^{(N-1)}$ is again a potential with similar properties to $V_{I}^{(N-1)}$ and $R^{(N-1)}(\lambda)|I|$ is a remainder term.

2 Let's observe that only the even order cumulants become infinite in the limit $N \rightarrow \infty$ (see [1]) and therefore modifying the definition of the ultraviolet stability one can avoid the subtraction of the odd order cumulant terms 
Then $\tilde{V}_{I}^{(N-1)}$ is such that it is possible to iterate (1.8) obtaining, for a generic frequency $h$

$$
\int P\left(d \tilde{\varphi}^{(h)}\right) \exp \tilde{V}_{I}^{(h)}=\exp \left[\tilde{V}_{I}^{(h-1)}+R^{(h-1)}(\lambda)|I|\right],
$$

where

$$
\begin{aligned}
\tilde{V}_{I}^{(h-1)} & =\left[\sum_{1}^{n-1} \frac{1}{k !} \tilde{\mathscr{E}}_{h}^{T}\left(\tilde{V}_{I ; k}^{(h)}\right)\right]_{n-1}, \\
\tilde{V}_{I}^{(N)} & =V_{I}^{(N)} .
\end{aligned}
$$

Here $\tilde{\mathscr{E}}_{h}^{T}(\cdot ; k)$ is the truncated expectation of order $k$ with respect to the measure $P\left(d \tilde{\varphi}^{(h)}\right)$ and $\left[\sum_{k} c_{k} \lambda^{k}\right]_{n-1}$ is the truncation to order $n-1$ of the polynomial $\sum_{k} c_{k} \lambda^{k}$, and the remainder satisfies

$$
\left|R^{(h)}(\lambda)\right| \leqq C\left(\lambda \gamma^{\left(\frac{\alpha^{2}}{4 \pi}-2\right) h}\right)^{n} \gamma^{2 h} \equiv C\left(\lambda_{\text {eff }}^{(h)}\right)^{n} \gamma^{2 h}
$$

where $C$ is some constant $h$-independent.

After performing all the $N$ integrations we get

$$
\int P\left(d \varphi^{(N)} \exp V_{I}^{(N)}=\exp \left[\sum_{0}^{N} R^{(h-1)}(\lambda)|I|\right]\right.
$$

and, provided that $\sum_{0}^{\infty} R^{(h-1)}(\lambda)$ is finite we can, from (1.11), obtain immediately the ultraviolet stability inequalities.

Using (1.11) we see that

$$
\sum_{0}^{N} R^{(h-1)}(\lambda) \leqq \sum_{0}^{\infty} h\left|R^{(h-1)}(\lambda)\right| \leqq C \lambda^{n} \sum_{0}^{\infty} \gamma^{\left[\left(\frac{\alpha^{2}}{4 \pi}-2\right) n+2\right](h-1)},
$$

which is finite provided

$$
\alpha^{2}<8 \pi\left(1-\frac{1}{n}\right)=\alpha_{n}^{2}
$$

The main part of the proof consists therefore in proving (1.9) and (1.11); in the next sections we'll discuss in detail the problem arising in [1] for the upper bound and the way of getting rid of it.

\section{The Upper Bound}

To understand the way in which the spurious threshold is eliminated it is necessary to recall, in some detail, how it appeared in [1]. The careful discussion of the upper bound is in Sect. 5 of [1], (see also [2]); here we only recall the main strategy.

As we want an upper bound estimate we cannot put, ab initio, in the integral (1.9) any characteristic function to constrain the field to be Hölder-continuous. Nevertheless to obtain, performing the integration frequency by frequency, at each step, an interaction potential such that the condition (1.11) for the remainder is satisfied we have to exclude the regions where the fields are "rough" (not Höldercontinuous). For that purpose we define a potential $\hat{V}_{J}^{(h)}$, where for any choice of 
the field $\varphi^{(h)}$ we subtract the regions where $\varphi^{(h)}$ is rough (see formula (3.23) of [1]); these regions are $\varphi^{(h)}$-dependent, but to perform the integration with respect to $P\left(d \tilde{\varphi}^{(h)}\right)$ we have to avoid this complicated dependence of the potential on $\tilde{\varphi}^{(h)}$ through the integration regions; to do that one defines a different function $H_{J}^{(h)}$ which depends only on the field $\varphi^{(h-1)}$, via the integration regions, and whose dependence on $\tilde{\varphi}^{(h)}$ is of the same type as that of $\tilde{V}_{J}^{(h)}$ and therefore can be integrated respect to it.

This is significant because between $\tilde{V}_{J}^{(h)}, \hat{V}_{J}^{(h)}$, and $H_{J}^{(h)}$ there are the following relations

$$
\begin{aligned}
\tilde{V}_{J}^{(N-1)} & \leqq \hat{V}_{J}^{(N-1)} \\
\hat{V}_{J}^{(h)} & \leqq \bar{A} \lambda_{\text {eff }}^{(h)} B_{h}^{2} \gamma^{2 h}\left|\hat{R}_{h} \cap I\right|+H_{J \backslash \hat{R}_{h}}^{(h)}
\end{aligned}
$$

[ see Eqs. (5.3) and (5.4) of Lemma 2 of [1] for the definition of $\hat{R}_{h}$ which there is denoted by $\hat{R}_{h}$, which here indicates a different set (see Sect. 4 )]. Once these inequalities are proved the integration for the upper bound is reduced to the following one

$$
\int P\left(d \tilde{\varphi}^{(h)}\right) \chi_{R_{h}^{c}}^{B_{h}} \overbrace{R_{h}}^{B_{h}} \exp H_{J \backslash \hat{R}_{h}}^{(h)}
$$

(see Eq. (3.32) of [1] and Eqs. (4.21) and (4.22) of [2]), and we can apply Lemma 1 of [1], obtaining, apart from a remaining term with the right properties,

$$
\exp \left[\sum_{0}^{n-1} \frac{1}{k !} \tilde{\mathscr{E}}_{h}^{T}\left(H_{J}^{(h)} ; k\right)\right]_{n-1} .
$$

To produce an iteration mechanism we have to recover $\hat{V}_{J}^{(h-1)}$ from

$$
\left[\sum_{0}^{n-1} \frac{1}{k !} \tilde{\mathscr{E}}_{h}^{T}\left(H_{J}^{(h)} ; k\right)\right]_{n-1}
$$

and this was only partially provided from Eq. (5.5) of [1] of Lemma 2 as this relation was valid only for $\alpha^{2}<\bar{\alpha}^{2}$. Therefore what we have to do is to devise a mechanism to avoid the need of inequality (5.5) of [1] in the proof of the upper bound. This is the content of the next two sections.

3.

Let's assume $\alpha^{2} \in\left[6 \pi, \frac{32}{5} \pi\right)$; in this interval the cumulant expansion has to be performed until the fourth order. At a generic level $h$, we have (see Eq. (3.1) of [1] $)^{3}$

$$
\begin{aligned}
\tilde{V}_{I}^{(h-1)} & =\left[\sum_{1}^{4} \frac{1}{k !} \tilde{\mathscr{E}}_{h}^{T}\left(\tilde{V}_{I}^{(h)} ; k\right)\right]_{4} \\
& =V_{0, I}^{(h-1)}-W_{I^{2}}^{(2, h-1)}+\sum_{1}^{2} W_{I^{3}}^{(3, i, h-1)}+\sum_{1}^{7} W_{I^{2} \times I^{2}}^{(4, i, h-1)}+A_{I}^{(h-1)}+C_{I}^{(h-1)},
\end{aligned}
$$

where the explicit expressions for all these terms are written in Eqs. (3.2)-(3.13) of [1]. As we discussed in Sect. 2 (see [1, Sect. 3] for more details) to obtain a

3 The factors $\lambda_{h}^{(k)}(\mathrm{k}: 2,3,4)$ appearing in Eq. (3.1) of [1] have been included in the definition of the $W$-terms $\left(\lambda_{h}=\gamma^{\alpha^{2} / 4 \pi} \lambda_{e f f}^{(h)}\right)$ 
remainder with the right properties [Eq. (1.11)] after having performed the integration with respect to the $P\left(d \tilde{\varphi}^{(h)}\right)$-measure we should need instead of $\tilde{V}_{I}^{(h)}$ an interaction $\hat{V}_{I}^{(h)}$ where the regions of $I \times I$, where $\varphi^{(h)}$ is not Hölder-continuous are subtracted. Then $\hat{V}_{I}^{(h)}$ has the following explicit expression, $\forall h$

$$
\begin{aligned}
\hat{V}_{I}^{(h)}= & V_{0, I}^{(h)}-W_{I^{2} \backslash \mathscr{D}_{h}}^{(2, h)}+\sum_{1}^{2} W_{I^{3}}^{(3, i, h)} \\
& +\sum_{3}^{5} W_{I^{2} \backslash \mathscr{D}_{h} \times I^{2} \backslash \mathscr{D}_{h}}^{(4,, i)}+W_{I^{2} \backslash \mathscr{D} h \times I^{2}}^{(4,1, h)} \\
& +W_{I^{2} \times I^{2} \backslash \mathscr{D}_{h}}^{(4,2, h)}+W_{I^{2} \backslash \mathscr{D}_{h} \times I^{2}}^{(4,6, h)}+W_{I^{2} \backslash \mathscr{D}_{h} \times I^{2}}^{(4,7, h)}+A_{I}^{(h)}+C_{I}^{(h)}
\end{aligned}
$$

Due to the complicated dependence on the field $\tilde{\varphi}^{(h)}$ through the regions $\mathscr{D}_{h}$ [see Eq. (3.21) of [1] and Eq. (4.1) of Sect. 4 for the definition of these regions] the integrations with respect to the $P\left(d \tilde{\varphi}^{(h)}\right)$-measure in the cumulant expansion cannot be explicitly performed anymore.

Therefore we are forced to introduce, using the relations (2.1) proved in [1], another function $H_{I}^{(h)}$ defined in the following way:

$$
\begin{aligned}
H_{I}^{(h)}= & V_{0, I}^{(h)}-W_{I^{2} \backslash \mathscr{D}_{h-1}}^{(2, h)}+\sum_{1}^{2} W_{I^{3}}^{(3, i, h)}+\sum_{3}^{5} W_{I^{2} \backslash \mathscr{D}_{h-1} \times I^{2} \backslash \mathscr{D}_{h-1}}^{(4, i, h)} \\
& +W_{I^{2} \backslash \mathscr{D}_{h-1} \times I^{2}}^{(4,1, h)}+W_{I^{2} \times I^{2} \backslash \mathscr{D}_{h-1}}^{(4,2, h)}+\sum_{6}^{7} W_{I^{2} \backslash \mathscr{D}_{h-1} \times I^{2}}^{(4, i, h)} \\
& +A_{I}^{(h)}+C_{I}^{(h)},
\end{aligned}
$$

which does not depend on $\tilde{\varphi}^{(h)}$ through the subtracted region $\mathscr{D}_{h-1}$ and whose cumulants can be computed explicitly.

Performing the integration $\int P\left(d \tilde{\varphi}^{(h)}\right) \chi_{R_{h}}^{B_{h}} \exp H_{I \backslash \hat{\kappa}_{h}}^{(h)}$ (see Sect. 4) we are left with

$$
\exp \hat{V}_{I}^{(h-1)}=\exp \left[\sum_{1}^{4} \frac{1}{k !} \tilde{\mathscr{E}}_{h}^{T}\left(H_{I}^{(h)} ; k\right)\right]_{4},
$$

plus some terms which will be included in the remainder (see Eq. (3.32) of [1] and Sect. 4). Then $\hat{V}_{I}^{(h-1)}$ has to be connected in the right way to $\hat{V}_{I}^{(h-1)}$ so that the procedure can be iterated.

From Appendix B of [1, Eqs. (B.1)-(B.11)] we have

$$
\begin{aligned}
\hat{V}_{I}^{(h-1)}-\hat{V}_{I}^{(h-1)}= & {\left[\sum_{1}^{4} \frac{1}{k !} \tilde{\mathscr{E}}_{h}^{T}\left(H_{I}^{(h)} ; k\right)\right]_{4}-\hat{V}_{I}^{(h-1)} } \\
= & -\bar{W}_{2}^{(h-1)}\left(\mathscr{D}_{h-1}\right)+\bar{W}_{4}^{(h-1)}\left(\mathscr{D}_{h-1}\right)+\bar{C}^{(h-1)}\left(\mathscr{D}_{h-1}\right) \\
& +\sum_{1}^{4} T_{i}^{(h-1)}\left(\mathscr{D}_{h-1}\right) .
\end{aligned}
$$

In [1] the idea was to use the positivity of the $\bar{W}_{2}^{(h-1)}\left(\mathscr{D}_{h-1}\right)$ term plus the fact that it is of second order in the effective coupling constant $\left(\lambda_{\text {eff }}^{(h)} \rightarrow 0\right.$ as $h \rightarrow \infty$, for $\left.\alpha^{2}<8 \pi\right)$ to dominate with it the other terms of (3.5) which are of order $\left(\lambda_{\text {eff }}^{(h)}\right)^{4}$ proving that (3.5) was negative and therefore could be thrown away. Unfortunately a detailed investigation of their explicit structure showed that it was impossible to prove completely that (3.5) was negative; then we decomposed the fourth order terms in 
two parts one which was dominated by $\bar{W}_{2}^{(h-1)}\left(\mathscr{D}_{h-1}\right)$ and the other which, as summable in $h$, could be safely put in the remainder. The way this was done implied an upper bound for the allowed $\alpha$ values : $\alpha^{2}>\bar{\alpha}^{2}$ (see Eqs. (B.13)-(B.22) of [1]). To remove this upper bound on $\alpha^{2}$ is the main goal of this paper which is obtained by Theorem 1 of Sect. 4.

We follow this strategy: we use the positivity of $\bar{W}_{2}^{(h-1)}\left(\mathscr{D}_{h-1}\right)$ only to control those terms $\left(\sum_{i}^{4} T_{i}^{(h-1)}\left(\mathscr{D}_{h-1}\right)\right)$ which can be dominated by it without any restriction on $\alpha^{2}$; therefore we are left with the problem of dealing with the terms

$$
\bar{W}_{4}^{(h-1)}\left(\mathscr{D}_{h-1}\right)+\bar{C}^{(h-1)}\left(\mathscr{D}_{h-1}\right) .
$$

First of all, let's observe that we can bound these terms, which are integrals of the following kind

$$
\int_{J^{2} \times \mathscr{D}_{h-1}} d \xi_{1} \ldots d \xi_{4} F\left(\varphi^{(h-1)} ; N, h\right)
$$

substituting the integrands with their modulus and eliminating their dependence on $\left(e^{i \alpha \underline{\varepsilon} \underline{\varphi}^{(h-1)}}-1\right)$, where it appears, (after having removed the Wick-dots) majorizing it by 2 .

Therefore we get

$$
\left(\bar{W}_{4}^{(h-1)}\left(\mathscr{D}_{h-1}\right)+\bar{C}^{(h-1)}\left(\mathscr{D}_{h-1}\right)\right) \leqq \tilde{C}_{I^{2} \times \mathscr{D}_{h-1}}^{(h-1)},
$$

and now $\tilde{C}_{I^{2} \times \mathscr{Q}_{h-1}}^{(h-1)}$ depends on $\varphi^{(h-1)}$ only through $\mathscr{D}_{h-1}$.

Using the explicit expression (B.3)-(B.9) written in [1] it is easy to prove that

$$
\tilde{C}_{I^{2} \times \mathscr{D}_{h}}^{(h)} \leqq A\left(\lambda_{\text {eff }}^{(h)}\right)^{4} \gamma^{2 h}\left|\Omega\left(\mathscr{D}_{h}\right)\right|,
$$

where

$$
\left|\Omega\left(\mathscr{D}_{h}\right)\right| \equiv \int_{\left(\xi_{1}, \xi_{2}\right) \in \mathscr{D}_{h}} d\left(\xi_{1}+\xi_{2}\right)
$$

and $A$ is an $h, N$-independent constant. Therefore for any $h$ we can write

$$
\hat{V}_{I}^{(h)} \leqq \hat{V}_{I}^{(h)}+\tilde{C}_{I^{2} \times \mathscr{D}_{h}}^{(h)},
$$

where $\tilde{C}_{I^{2} \times \mathscr{D}_{h}}^{(h)}$ satisfies (3.9).

In the next section we discuss how to accomplish our goal, that is how to put the $\tilde{C}_{I^{2} \times \mathscr{D}_{h}}^{(h)}$-contributions, after the integration, in the remainder.

\section{The Main Result}

Let's start with some definitions

$$
\begin{aligned}
\mathscr{D}_{h}\left(\varphi^{(h)}\right)= & \left\{(\xi, \eta) \in I^{2}|| \sin \frac{\alpha}{2}\left(\varphi_{\xi}^{(h)}-\varphi_{\eta}^{(h)}\right) \mid \geqq B_{h}\left(\gamma^{h}|\xi-\eta|\right)^{1-\varepsilon},\right. \\
& \left.B_{h}\left(\gamma^{h}|\xi-\eta|\right)^{1-\varepsilon} \leqq \delta<1\right\}, \\
R_{h}\left(\tilde{\varphi}^{(h)}\right)= & \left\{\Delta \in Q_{h} \mid \exists \xi, \eta \in \Delta, \text { such that }\left|\sin \frac{\alpha}{2}\left(\tilde{\varphi}_{\zeta}^{(h)}-\tilde{\varphi}_{\eta}^{(h)}\right)\right|\right. \\
\geqq & \left.\bar{B}_{h}\left(\gamma^{h}|\xi-\eta|\right)^{1-\varepsilon}\left(1+\gamma^{h} d(\Delta, I)\right)\right\},
\end{aligned}
$$


with

$$
\begin{aligned}
B_{h} & =B \log \left(e+\lambda^{-1}\right)\left(1+h^{3}\right), \\
\bar{B}_{h} & =\bar{B} \log \left(e+\lambda^{-1}\right)\left(1+h^{2}\right), \\
\hat{R}_{h}\left(\tilde{\varphi}^{(h)}\right) & =\left\{\Delta \in Q_{h} \mid \gamma^{h} d\left(\Delta, R_{h}\left(\tilde{\varphi}^{(h)}\right)\right) \leqq 1\right\} .{ }^{4}
\end{aligned}
$$

From these definitions the following lemma follows easily

Lemma 1. Let $\bar{B}=\frac{\sigma^{-1}}{2} B$ with $0<\sigma^{-1} \leqq 1$ and $B>1$ then

$$
\left(\mathscr{D}_{h} \backslash \mathscr{D}_{h-1}\right)\left(\varphi^{(h)}\right) \subseteq\left(\hat{R}_{h} \times \hat{R}_{h}\right)\left(\tilde{\varphi}^{(h)}\right) .
$$

Proof. The proof (trivial) uses the following "triangular" inequality

$$
\left|\sin \frac{\alpha}{2}\left(\tilde{\varphi}_{\xi}^{(h)}-\tilde{\varphi}_{\eta}^{(h)}\right)\right|+\left|\sin \frac{\alpha}{2}\left(\varphi_{\xi}^{(h-1)}-\varphi_{\eta}^{(h-1)}\right)\right| \geqq\left|\sin \frac{\alpha}{2}\left(\varphi_{\xi}^{(h)}-\varphi_{\eta}^{(h)}\right)\right|
$$

and the observation that if $(\xi, \eta) \in \mathscr{D}_{h}$ then

$$
\left(\gamma^{h}|\xi-\eta|\right) \leqq\left(\delta B_{h}^{-1}\right)^{\frac{1}{1-\varepsilon}}<1
$$

which implies that or $\xi$ and $\eta$ belong to the same $\Delta \in Q_{n}$ or they belong to two adjacent ones.

From Lemma 1 we have

$$
\begin{aligned}
\mathscr{D}_{h}\left(\varphi^{(h)}\right) & =\left(\mathscr{D}_{h} \backslash \mathscr{D}_{h-1}\right)\left(\varphi^{(h)}\right) \cup\left(\mathscr{D}_{h} \cap \mathscr{D}_{h-1}\right)\left(\varphi^{(h)}\right) \\
& \cong\left(\hat{R}_{h} \times \hat{R}_{h}\right)\left(\tilde{\varphi}^{(h)}\right) \cup\left(\left(\mathscr{D}_{h} \cap \mathscr{D}_{h-1}\right) \backslash\left(\hat{R}_{h} \times \hat{R}_{h}\right)\right)\left(\varphi^{(h)}\right) .
\end{aligned}
$$

Let's now investigate the second region of this inclusion. We have the following result :

\section{Lemma 2.}

$$
\left(\left(\mathscr{D}_{h} \cap \mathscr{D}_{h-1}\right) \backslash\left(\hat{R}_{h} \times \hat{R}_{h}\right)\right)\left(\varphi^{(h)}\right) \subseteq \mathscr{D}_{h-1}\left(B_{(h, h-1)}\right)\left(\varphi^{(h-1)}\right)
$$

where

$$
B_{(h, h-1)}=\frac{B\left(1+h^{3}\right)}{\left(1+(h-1)^{3}\right)} \gamma^{1-\varepsilon^{\prime}} .
$$

Then $\mathscr{D}_{h-1}\left(B_{(h, h-1)}\right)$ has the same definition as in (4.1) with $B$ substituted by $B_{(h, h-1)}$ and $\varepsilon^{\prime}$ will be defined during the proof of the lemma.

Proof. Let $(\xi, \eta)$ be a couple of points belonging to $\left(\mathscr{D}_{h} \cap \mathscr{D}_{h-1}\right) \backslash \hat{R}_{h} \times \hat{R}_{h}$. Then the following inequalities hold

a)

$$
\begin{aligned}
& B_{h}\left(\gamma^{h}|\xi-\eta|\right)^{1-\varepsilon} \leqq \delta \\
& \left|\sin \frac{\alpha}{2}\left(\varphi_{\xi}^{(h)}-\varphi_{\eta}^{(h)}\right)\right| \geqq B_{h}\left(\gamma^{h}|\xi-\eta|\right)^{1-\varepsilon}, \\
& \left|\sin \frac{\alpha}{2}\left(\tilde{\varphi}_{\xi}^{(h)}-\tilde{\varphi}_{\eta}^{(h)}\right)\right|<2 \bar{B}_{h}\left(\gamma^{h}|\xi-\eta|\right)^{1-\varepsilon}, \\
& \left|\sin \frac{\alpha}{2}\left(\varphi_{\xi}^{(h-1)}-\varphi_{\eta}^{(h-1)}\right)\right| \leqq B_{h-1}\left(\gamma^{h-1}|\xi-\eta|\right)^{1-\varepsilon}
\end{aligned}
$$

b)

c)

d)

$4 Q_{h}$ is a pavement of $R^{2}$ made by cubic tesserae with side size $\gamma^{-h} ; d(\Delta, F)$ is the distance between $\Delta$ and the region $F$ 
The relation d) can be strengthened; in fact using the "triangular" inequality

$$
\begin{aligned}
\left|\sin \frac{\alpha}{2}\left(\varphi_{\xi}^{(h-1)}-\varphi_{\eta}^{(h-1)}\right)\right| & \geqq\left|\sin \frac{\alpha}{2}\left(\varphi_{\xi}^{(h)}-\varphi_{\eta}^{(h)}\right)\right|-\left|\sin \frac{\alpha}{2}\left(\tilde{\varphi}_{\xi}^{(h)}-\tilde{\varphi}_{\eta}^{(h)}\right)\right| \\
& \geqq\left(B_{h}-2 \bar{B}_{h}\right) \gamma^{(1-\varepsilon)}\left(\gamma^{h-1}|\xi-\eta|\right)^{1-\varepsilon},
\end{aligned}
$$

then

$$
B_{h}-2 \bar{B}_{h}=\left(\text { choosing } \sigma^{-1}=1 \text { and therefore } \bar{B}=B / 2\right)=B\left(h^{3}-h^{2}\right)>B \gamma^{-\tilde{\delta}}\left(1+h^{3}\right),
$$

where $\tilde{\delta}$ is a monotone decreasing function of $h$. Therefore defining $\varepsilon^{\prime}=\varepsilon+\tilde{\delta}$ we get

$$
\begin{aligned}
& \left(\left(\mathscr{D}_{h} \cap \mathscr{D}_{h-1}\right) \backslash \hat{R}_{h} \times \hat{R}_{h}\right)\left(\varphi^{(h)}\right) \subseteq\left\{(\xi, \eta) \in I^{2}|| \sin \frac{\alpha}{2}\left(\varphi_{\xi}^{(h-1)}-\varphi_{\eta}^{(h-1)}\right) \mid\right. \\
& \geqq B_{(h, h-1)}\left(1+(h-1)^{3}\right)\left(\gamma^{h-1}|\xi-\eta|\right)^{1-\varepsilon} ; \\
& \left.\quad B_{(h, h-1)}\left(1+(h-1)^{3}\right)\left(\gamma^{h-1}|\xi-\eta|\right)^{1-\varepsilon} \leqq \delta\right\}=\mathscr{D}_{h-1}\left(B_{(h, h-1)}\right)\left(\varphi^{(h-1)}\right) .
\end{aligned}
$$

From Lemmas 1 and 2 we get, omitting the field dependence

$$
\mathscr{D}_{h}(B) \subseteq\left(\hat{R}_{h} \times \hat{R}_{h}\right)(B) \cup \mathscr{D}_{h-1}\left(B_{(h, h-1)}\right) .
$$

As the proof of Lemmas 1 and 2 does not depend on the choice of $B$ we can iterate the procedure obtaining

$$
\mathscr{D}_{h-1}\left(B_{(h, h-1)}\right) \subset\left(\hat{R}_{h-1} \times \hat{R}_{h-1}\right)\left(B_{(h, h-1)}\right) \cup \mathscr{D}_{h-2}\left(B_{(h, h-2)}\right),
$$

where

$$
B_{(h, h-2)}=B \gamma^{2\left(1-\varepsilon^{\prime}\right)} \frac{\left(1+h^{3}\right)}{\left(1+(h-2)^{3}\right)} .
$$

Repeating the procedure until $h=0$ we get

$$
\mathscr{D}_{h}\left(B ; \varphi^{(h)}\right) \leqq \bigcup_{0}^{h}\left(\hat{R}_{k} \times \hat{R}_{k}\right)\left(B_{(h, k)} ; \tilde{\varphi}^{(k)}\right)
$$

with an obvious change of notations, where

$$
B_{(h, k)}=B \gamma^{(h-k)\left(1-\varepsilon^{\prime}\right)} \frac{\left(1+h^{3}\right)}{\left(1+(h-k)^{3}\right)} .
$$

As, see Eq. (3.7),

$$
\tilde{C}_{I^{2} \times \mathscr{D}_{h}}^{(h)}=\int_{I^{2} \times \mathscr{D}_{h}} d \xi|F(N, h ; \xi)|,
$$

we can write

$$
\tilde{C}_{I^{2} \times \mathscr{D}_{h}\left(B, \varphi^{(h)}\right)}^{(h)} \leqq \sum_{0}^{h} \tilde{C}_{I^{2} \times\left(\hat{R}^{k}\right)^{2}\left(B_{(h, k)} ; \tilde{\varphi}^{(k)}\right)},
$$

and, of course, from (3.8)

$$
\tilde{C}_{I^{2} \times\left(\hat{R}_{k}\right)^{2}\left(B_{(h, k)} ; \tilde{\varphi}^{(k)}\right)} \leqq A^{\prime}\left(\lambda_{e f f}^{(h)}\right)^{4} \gamma^{2 h}\left|\hat{R}_{k}\left(B_{(h, k)} ; \tilde{\varphi}^{(h)}\right)\right|
$$


with some constant $A^{\prime}$ independent of $h$ and $N$. Remembering Eq. (3.9) we have, writing $N$ instead of $N-2$ for notational simplicity,

$$
\begin{aligned}
\hat{V}_{I}^{(N)} & \leqq \hat{V}_{I}^{(N)}+\tilde{C}_{I^{2} \times \mathscr{D}_{N}\left(B ; \varphi^{(N)}\right)}^{(N)} \leqq \hat{V}_{I}^{(N)}+\sum_{0}^{N} \tilde{C}_{I^{2} \times\left(\hat{R}_{k}\right)^{2}\left(B_{(N, k)} ; \tilde{\varphi}^{(k)}\right)}^{(N)} \\
& =\left(\hat{V}_{I}^{(N)}+\tilde{C}_{I^{2} \times\left(\hat{R}_{N}\right)^{2}\left(B, \tilde{\varphi}^{(N))}\right.}^{(N)}\right)+\sum_{0}^{N-1} \tilde{C}_{I^{2} \times\left(\hat{R}_{k}\right)^{2}\left(B_{(N, k)} ; \tilde{\varphi}^{(k)}\right)}^{(N)}
\end{aligned}
$$
and with respect to the $P\left(d \tilde{\varphi}^{(N)}\right)$ integration the $\sum_{0}^{N-1} \ldots$ part is a true constant as it
does not depend on $\tilde{\varphi}^{(N)}$.

At this stage, therefore, we have only to show that, after the integration is performed we can safely put the contribution coming from $\tilde{C}_{I^{2} \times\left(\hat{R}_{N}\right)^{2}\left(B ; \tilde{\varphi}^{(N)}\right)}$ in the remainder. Let's suppose that this can be done, (this will be proven in a general way later) in this case we are left with

$$
\begin{aligned}
& \hat{\hat{V}}_{I}^{(N-1)} \equiv \hat{V}_{I}^{(N-1)}+\sum_{0}^{N-1} \tilde{C}_{I^{2} \times\left(\hat{R}_{k}\right)^{2}\left(B_{(N, k)} ; \tilde{\varphi}^{(k)}\right)}^{N} \\
& \leqq \hat{V}_{I}^{(N-1)}+\tilde{C}_{I^{2} \times \mathscr{D}_{N-1}\left(B, \varphi^{(N-1)}\right)}^{(N-1)}+\sum_{0}^{N-1} \tilde{C}_{I^{2} \times\left(\hat{R}_{k}\right)^{2}\left(B_{(N, k)} ; \tilde{\varphi}^{(k)}\right)}^{(N)} \\
& \leqq\left(\hat{V}_{I}^{(N-1)}+\tilde{C}_{I^{2} \times\left(\hat{R}_{N-1}\right)^{2}\left(B_{(N, N-1)}^{(N)}, \tilde{\varphi}^{(N-1))}\right.}+\tilde{C}_{I^{2} \times\left(\hat{R}_{N-1}\right)^{2}\left(B_{(N-1, N-1)}^{(N-1)}, \tilde{\varphi}^{(N-1)}\right)}^{)}\right. \\
& +\sum_{0}^{N-2}\left(\tilde{C}_{I^{2} \times\left(\hat{R}_{k}\right)^{2}\left(B_{(N, k)} ; \tilde{\varphi}^{(k)}\right)}^{(N)}+\tilde{C}_{I^{2} \times\left(\tilde{R}_{k}\right)^{2}\left(B_{(N-1, k)}^{(N-1)} ; \tilde{\varphi}^{(k))}\right)}\right),
\end{aligned}
$$

and again the first parentheses is the only $\tilde{\varphi}^{(N-1)}$ dependent part.

At level $h$ we have

$$
\begin{aligned}
\hat{\hat{V}}_{I}^{(h)} \leqq & \left(\hat{V}_{I}^{(h)}+\sum_{h}^{N} \tilde{C}_{I^{2} \times\left(\hat{R}_{h}\right)^{2}\left(B_{(q, h)}^{(q)} ; \tilde{\varphi}^{(h)}\right)}\right) \\
& \left.+\sum_{0}^{h-1} \sum_{h}^{N} \tilde{C}_{I^{2} \times\left(\hat{R}_{k}\right)^{2}\left(B_{(q, k)} ; \tilde{\varphi}^{(k)}\right)}\right) .
\end{aligned}
$$

Therefore to show that the iterative mechanism can be performed we have to prove the following theorem

\section{Theorem 1.}

$$
\begin{aligned}
\int & P\left(d \tilde{\varphi}^{(h)}\right) \exp \left[\hat{V}_{I}^{(h)}+\sum_{h}^{N} \tilde{C}_{I^{2} \times\left(\hat{R}_{h}\right)^{2}\left(B_{(q, h)} ; \tilde{\varphi}^{(h)}\right)}^{(q)}\right] \\
\leqq & \exp c|I| \exp \left[\hat{V}_{I}^{(h-1)}+\tilde{C}_{I^{2} \times \mathscr{D}_{h-1}\left(B, \varphi^{(h-1)}\right)}^{(h-1)}\right]
\end{aligned}
$$

for some constant $c$ independent of $h$ and $N$.

Proof. The proof is based on a slightly more refined version of the Lemma 3 of [2] (the "tail lemma"); Let's start by introducing some simpler notations:

Let $h$ be a fixed, but arbitrary, frequency, then

$$
\begin{aligned}
R_{h}\left(B_{(q, h)} ; \tilde{\varphi}^{(h)}\right) & \equiv R_{q}\left(\tilde{\varphi}^{(h)}\right), \\
B_{(q, h), h} & \equiv B_{(q, h)} \log \left(e+\lambda^{-1}\right)\left(1+h^{3}\right) \equiv B_{q}, \\
\bar{B}_{(q, h), h} & \equiv \frac{1}{2} B_{(q, h)} \log \left(e+\lambda^{-1}\right)\left(1+h^{2}\right) \equiv \bar{B}_{q}, \\
\tilde{C}_{I^{2} \times\left(\hat{R}_{q}\right)^{2}\left(\tilde{\varphi}^{(h)}\right)}^{(q)} & \leqq A^{\prime \prime}\left(\lambda_{\text {eff }}^{(q)}\right)^{4} \gamma^{2 q}\left|R_{q}\right|\left(\tilde{\varphi}^{(h)}\right)
\end{aligned}
$$


for some constant $A^{\prime \prime}$ independent of $q$ and $N$, and of course, assuming $I$ a region exactly paved by $\Delta$ 's $\in Q_{0}$, it follows that

$$
R_{q}\left(\tilde{\varphi}^{(h)}\right)=R_{q}\left(\tilde{\varphi}^{(h)}\right) \cap I .
$$

Let's consider the following decomposition of the identity

$$
\begin{gathered}
1=\sum_{G_{h} \backslash G_{h+1}} \sum_{G_{h+1} \backslash G_{h+2}} \ldots \sum_{G_{N-1} \backslash G_{N}} \sum_{G_{N}} \chi_{Q_{h} \backslash G_{h}}^{\bar{B}_{h}} \dot{\chi}_{G_{h} \backslash G_{h+1}}^{\bar{B}_{h}} \ldots \dot{\chi}_{G_{N-1} \backslash G_{N}}^{\bar{B}_{N-1}} \dot{\chi}_{G_{N}}^{\bar{B}_{N}} \\
\dot{\chi}_{G_{k} \backslash G_{k+1}} \equiv \prod_{\Delta \in G_{k} \backslash G_{k+1}} \dot{\chi}_{\Delta}^{\bar{B}_{k}},
\end{gathered}
$$

where

$$
\grave{\chi}_{\Delta}^{\bar{B}_{k}}=1-\chi_{\Delta}^{\bar{B}_{k}}
$$

and $\chi_{\Delta}^{\bar{B}_{k}}$ is the characteristic function of the $P\left(d \tilde{\varphi}^{(h)}\right)$-measurable event

$$
E_{\Delta}^{\bar{B}_{k}}=\left\{\tilde{\varphi}^{(h)} \mid \sup _{\xi, \eta \in \Delta} \frac{\left|\tilde{\varphi}_{\xi}^{(h)}-\tilde{\varphi}_{\eta}^{(h)}\right|}{\left(\gamma^{h}|\xi-\eta|\right)^{1-\varepsilon}} \leqq \bar{B}_{k}\left(1+\gamma^{h} d(\Delta, I)\right)\right\} .
$$

Let's remark that in (4.23) $G_{h}, G_{h+1}, \ldots, G_{N}$ are arbitrary sets of tesserae $\in Q_{h}$ and do not depend on $\tilde{\varphi}^{(h)}$. Moreover $G_{h} \supset G_{n+1} \supset \ldots \supset G_{N}$ and

$$
G_{h}=\bigcup_{h}^{N} G_{k} \backslash G_{k+1} \quad\left(G_{N+1} \equiv \Phi\right),
$$

therefore

$$
\begin{aligned}
& {[I] \equiv \int P\left(d \tilde{\varphi}^{(h)}\right) \exp \left[\hat{V}_{I}^{(h)}+\sum_{h}^{N} \tilde{C}_{I^{2} \times \hat{R}_{q}^{2}\left(\tilde{\varphi}^{(h)}\right)}^{(h)}\right]} \\
& =\sum_{\left(G_{h} \backslash G_{h+1}, \ldots, G_{N-1} \backslash G_{N}, G_{N}\right)} \int \exp \hat{V}_{I}^{(h)} \chi_{Q_{h} \backslash G_{h}}^{\bar{B}_{h}} \\
& \cdot \exp \sum_{h}^{N} \tilde{C}_{I^{2} \times \hat{R}_{q}^{2}\left(\tilde{\varphi}^{(h)}\right)}^{(q)} \dot{\chi}_{G_{h} \backslash G_{h+1}}^{\bar{B}_{h}} \ldots \dot{\chi}_{G_{N-1} \backslash G_{N}}^{\bar{B}_{N-1}} \dot{\chi}_{G_{N}}^{\bar{B}_{N}} P\left(d \tilde{\varphi}^{(h)}\right) \\
& \leqq \text { [using Eq. (2.1)] } \\
& \leqq \sum_{\left(G_{h} \backslash G_{h+1}, \ldots, G_{N} \backslash \backslash G_{N}, G_{N}\right)} \int P\left(d \tilde{\varphi}^{(h)}\right) \exp H_{I \backslash \hat{G}_{h}}^{(h)} \chi_{Q_{h} \backslash G_{h}}^{\bar{B}_{h}} \\
& \cdot\left\{\exp \left[\bar{A} \lambda_{\text {eff }}^{(h)} B_{h}^{2} \gamma^{2 h}\left|\hat{G}_{h} \cap I\right|+\sum_{h}^{N} \tilde{C}_{I^{2} \times \hat{R}_{q}^{2}\left(\tilde{\varphi}^{(h)}\right)}^{(q)}\right]\right. \\
& \left.\cdot \chi_{G_{h} \backslash G_{h+1}}^{\bar{B}_{h}} \ldots \chi_{G_{G_{N-1} \backslash G_{N}}^{\bar{B}_{N-1}}} \chi_{G_{G_{N}}}^{\bar{B}_{N}}\right\} \text {. }
\end{aligned}
$$

Remembering Eq. (4.21) we have

$$
\begin{aligned}
\{(4.26)\} \leqq & \left(\dot{\chi}_{G_{h} \backslash G_{h+1}}^{\bar{B}_{h}} \ldots \dot{\chi}_{G_{N}}^{\bar{B}_{N}}\right) \exp \bar{A} \lambda_{\text {eff }}^{(h)} B_{h}^{2} \gamma^{2 h}\left|\hat{G}_{h} \cap I\right| \\
& \cdot \exp \left[\sum_{h}^{N} A^{\prime \prime}\left(\lambda_{\text {eff }}^{(q)}\right)^{4} \gamma^{2 q}\left|G_{N}\right|+\sum_{h}^{N-1} A^{\prime \prime}\left(\lambda_{\text {eff }}^{(q)}\right)^{4} \gamma^{2 q}\left|G_{N-1} \backslash G_{N}\right|\right. \\
& \left.\ldots+\sum_{h}^{h+1} A^{\prime \prime}\left(\lambda_{\text {eff }}^{(q)}\right)^{4} \gamma^{2 q}\left|G_{h+1} \backslash G_{h+2}\right|+A^{\prime \prime}\left(\lambda_{\text {eff }}^{(h)}\right)^{4} \gamma^{2 h}\left|G_{h} \backslash G_{h+1}\right|\right],
\end{aligned}
$$


where the idea is that, given $\tilde{\varphi}^{(h)}$, such that $\left(\dot{\chi}_{G_{h} \backslash G_{h+1}}^{\bar{B}_{h}} \ldots \dot{\chi}_{G_{N}}^{\bar{B}_{N}}\right)\left(\tilde{\varphi}^{(h)}\right)=1$ then contributions to the term proportional to $\left|G_{N}\right|$ can come from any $\tilde{C}_{I^{2} \times \hat{R}_{q}^{2}\left(\tilde{\varphi}^{(h)}\right)}$ with $q \in[h, N]$, but contributions to the terms proportional to $\left|G_{N-1} \backslash G_{N}\right|$ can come only from those $\tilde{C}_{I^{2} \times \hat{R}_{q}^{2}\left(\tilde{\varphi}_{(h)}^{(h)}\right)}^{(q)}$ with $q \in[h, N-1]$ and so on.

As $\left(\lambda_{\text {eff }}^{(q)}\right)^{4} \gamma^{2 q} \propto \gamma^{\left(\frac{\alpha^{2}}{\pi}-6\right) q} \underset{q \rightarrow \infty}{\longrightarrow} \infty$ we can bound each sum in the exponent by

$$
\sum_{h}^{k} A^{\prime \prime}\left(\lambda_{\text {eff }}^{(q)}\right)^{4} \gamma^{2 q}\left|G_{k} \backslash G_{k+1}\right| \leqq A^{\prime \prime}(k+1-h)\left(\lambda_{\text {eff }}^{(k)}\right)^{4} \gamma^{2 k}\left|G_{k} \backslash G_{k+1}\right|
$$

so that

$$
\begin{aligned}
\{(4.26)\} \leqq & \left(\dot{\chi}_{G_{h} \backslash G_{h+1}}^{\vec{B}_{h}} \ldots \dot{\chi}_{G_{N}}^{\vec{B}_{N}}\right) \exp \bar{A} \lambda_{\text {eff }}^{(h)} B_{h}^{2} \gamma^{2 h}\left|\hat{G}_{h} \cap I\right| \\
& \cdot \exp A^{\prime \prime}\left[(N+1-h)\left(\lambda_{\text {eff }}^{(N)}\right)^{4} \gamma^{2 N}\left|G_{N}\right|+(N-h)\left(\lambda_{\text {eff }}^{(N-1)}\right)^{4} \gamma^{2(N-1)}\left|G_{N-1} \backslash G_{N}\right|\right. \\
& \left.\ldots+2\left(\lambda_{\text {eff }}^{(h+1)}\right)^{4} \gamma^{2(h+1)}\left|G_{h+1} \backslash G_{h+2}+\left(\lambda_{\text {eff }}^{(h)}\right)^{4} \gamma^{2 h}\right| G_{h} \backslash G_{h+1} \mid\right] .
\end{aligned}
$$

Applying the main lemma (Lemma 1 of [1]) and the estimate (4.29) we get

$$
\begin{aligned}
{[I] \leqq } & \exp \left[\delta\left(B_{h}, \lambda_{e f f}^{(h)}\right) \gamma^{2 h}|I|\right] \exp \left[\sum_{1}^{4} \frac{1}{k !} \tilde{E}_{h}^{T}\left(H_{I}^{(h)} ; k\right)\right] \\
& \cdot\left\{\sum _ { ( G _ { h } \backslash G _ { h + 1 } \ldots G _ { N } ) } \operatorname { e x p } \left[\overline{\bar{A}} \lambda_{e f f}^{(h)} B_{h}^{2+\varrho} \gamma^{2 h}\left|\hat{\hat{G}}_{h} \cap I\right|\right.\right. \\
& \left.+A^{\prime \prime} \sum_{h}^{N}(q-h+1)\left(\lambda_{e f f}^{(q)}\right)^{4} \gamma^{2(q-h)} \gamma^{2 h}\left|G_{q} \backslash G_{q+1}\right|\right] \\
& \left.\cdot\left(\int P\left(d \tilde{\varphi}^{(h)}\right) \dot{\chi}_{G_{h} \backslash G_{h+1}}^{\bar{B}_{h}} \ldots \dot{\chi}_{G_{N-1} \bar{B}_{N}} \bar{B}_{N} \dot{\chi}_{G_{N}}^{\bar{B}_{N}}\right)^{1 / 2}\right\},
\end{aligned}
$$

where $\varrho$ is a positive number.

Remembering Eq. (3.9) and from [1] that $\delta\left(B_{h}, \lambda_{\text {eff }}^{(h)}\right) \gamma^{2 h} \leqq c^{\prime}$ which is $h$-independent we obtain the theorem, provided we prove that the \{\} of (4.30) satisfies the following inequality

$$
\{(4.30)\} \leqq e^{c^{\prime \prime}|I|}
$$

with some $h$ and $N$ independent constant.

Proof of (4.31). Applying the "tail lemma" (Proposition 1 of [1] and Lemma 3 of [2]) we get

$$
\begin{aligned}
& \left(\int P\left(d \tilde{\varphi}^{(h)}\right) \dot{\chi}_{G_{h} \backslash G_{h+1}}^{\bar{B}_{h}} \ldots \dot{\chi}_{G_{N-1} \backslash G_{N}}^{\bar{B}_{N-1}} \dot{\chi}_{G_{N}}^{\bar{B}_{N}}\right)^{1 / 2} \\
& \leqq \\
& \quad \prod_{\Delta \subset G_{h} \backslash G_{h+1}} \exp \frac{1}{2}\left(c_{1}-c_{2} \bar{B}_{h}^{2}\left(1+d\left(\Delta, \gamma^{2 h}|I|\right)\right)\right) \\
& \quad \cdot \prod_{\Delta \subset G_{h+1} \backslash G_{h+2}} \exp \frac{1}{2}\left(c_{1}-c_{2} \bar{B}_{h+1}^{2}\left(1+d\left(\Delta, \gamma^{2 h}|I|\right)\right)\right) \\
& \quad \ldots \prod_{\Delta \subset G_{N} \backslash 1 \backslash G_{N}} \exp \frac{1}{2}\left(c_{1}-c_{2} \bar{B}_{N-1}^{2}\left(1+d\left(\Delta, \gamma^{2 h}|I|\right)\right)\right) \\
& \quad \cdot \prod_{\Delta \subset G_{N}} \exp \frac{1}{2}\left(c_{1}-c_{2} \bar{B}_{N}^{2}\left(1+d\left(\Delta, \gamma^{2 h}|I|\right)\right)\right),
\end{aligned}
$$


which together with (4.29) gives

$$
\begin{aligned}
\{(4.30)\} \leqq & \sum_{\left(G_{h} \backslash G_{h+1} \ldots G_{N}\right)}\left(\prod _ { \Delta \subset G _ { h } \backslash G _ { h + 1 } } \operatorname { e x p } \left[A^{\prime \prime}\left(\lambda_{\text {eff }}^{(h)}\right)^{4}+\overline{\bar{A}} \lambda_{\text {eff }}^{(h)} B_{h}^{2+\varrho^{\prime}}+\frac{1}{2} c_{1}\right.\right. \\
& \left.-\frac{1}{2} c_{2} \bar{B}_{h}^{2}\left(1+d\left(\Delta, \gamma^{2 h} I\right)\right)\right] \ldots \prod_{\Delta \subset G_{q} \backslash G_{q+1}} \exp \left[A^{\prime \prime}\left(\lambda_{\text {eff }}^{(q)}\right)^{4} q \gamma^{2(q-h)}\right. \\
& \left.+\overline{\bar{A}} \lambda_{\text {eff }}^{(h)} B_{h}^{2+\varrho^{\prime}}+\frac{1}{2} c_{1}-\frac{1}{2} c_{2} \bar{B}_{q}^{2}\left(1+d\left(\Delta, \gamma^{2 h} I\right)\right)\right] \ldots \prod_{\Delta \subset G_{N}} \exp \left[A^{\prime \prime}\left(\lambda_{\text {eff }}^{(N)}\right)^{4}\right. \\
& \left.\left.\cdot N \gamma^{2(N-h)}+\overline{\bar{A}} \lambda_{\text {eff }}^{(h)} B_{h}^{2+\varrho^{\prime}}+\frac{1}{2} c_{1}-\frac{1}{2} c_{2} \bar{B}_{N}^{2}\left(1+d\left(\Delta, \gamma^{2 h} I\right)\right)\right]\right) \\
= & \prod_{\Delta \subset Q_{h}}\left(1+\sum_{h}^{N} \exp \left[A^{\prime \prime}\left(\lambda_{\text {eff }}^{(q)}\right)^{4} q \gamma^{2(q-h)}+\overline{\bar{A}} \lambda_{\text {eff }}^{(h)} B_{h}^{2+\varrho^{\prime}}\right.\right. \\
& \left.\left.+\frac{1}{2} c_{1}-\frac{1}{2} c_{2} \bar{B}_{q}^{2}\left(1+d\left(\Delta, \gamma^{2 h} I\right)\right)\right]\right)
\end{aligned}
$$

where $\varrho^{\prime}>\varrho$ and $\overline{\bar{A}}$ is some $N$-independent constant. From (4.20) and (4.13) it follows that

$$
\begin{aligned}
\bar{B}_{q}^{2} & =\frac{1}{4} B_{(q, h)}^{2}\left(\log \left(e+\lambda^{-1}\right)\right)^{2}\left(1+h^{2}\right)^{2} \\
& =\frac{1}{4} \frac{\left(1+q^{3}\right)^{2}}{\left(1+h^{3}\right)^{2}}\left(1+h^{2}\right)^{2}\left(\log \left(e+\lambda^{-1}\right)\right)^{2} B^{2} \gamma^{2(q-h)\left(1-\varepsilon^{\prime}\right)} \\
& \geqq c_{3} \bar{B}_{h}^{2} \gamma^{2(q-h)\left(1-\varepsilon^{\prime}\right)},
\end{aligned}
$$

where $c_{3}$ is a positive constant.

We have therefore for the exponent of the right hand side of (4.33)

$$
\begin{aligned}
{[(4.33)] \leqq } & {\left[A^{\prime \prime}\left(\lambda_{e f f}^{(q)}\right)^{4} q \gamma^{2(q-h)}+\overline{\bar{A}} \lambda_{e f f}^{(h)} B_{h}^{2+\varrho^{\prime}}\right.} \\
& \left.+\frac{1}{2} c_{1}-\frac{1}{2} c_{4} \bar{B}_{h}^{2} \gamma^{2(q-h)\left(1-\varepsilon^{\prime}\right)}\left(1+d\left(\Delta, \gamma^{2 h} I\right)\right)\right],
\end{aligned}
$$

where $c_{4}$ is some positive constant.

$$
\begin{gathered}
\text { As }\left(\lambda_{\text {eff }}^{(q)}\right)^{4}=\gamma^{\left(\frac{\alpha^{2}}{\pi}-8\right) q} \lambda^{4} \text {, it follows that for } 2 \varepsilon^{\prime}<\left(8-\frac{\alpha^{2}}{\pi}\right) \text { and for } q \gg h \\
{[(4.33)] \leqq-c_{5} \bar{B}_{h}^{2} \gamma^{2(q-h)\left(1-\varepsilon^{\prime}\right)}\left(1+d\left(\Delta, \gamma^{2 h} I\right)\right) \text { with } \quad c_{5}>0 .}
\end{gathered}
$$

Moreover defining $\varepsilon^{\prime}=\varepsilon+\tilde{\delta}$, fixed $\alpha^{2}$, it is possible to find $h_{1}\left(\alpha^{2}\right)$ such that for $q>h_{1}\left(\alpha^{2}\right)$

$$
2 \varepsilon^{\prime}<\left(8-\frac{\alpha^{2}}{\pi}\right) \text {. }
$$

Therefore if $h>h_{1}\left(\alpha^{2}\right)$, it follows that

$$
\sum_{h}^{N} q e^{[(4.33)]} \leqq \sum_{h}^{\infty} e^{[(4.33)]} \leqq e^{-c_{6} \bar{B}^{2}\left(1+d\left(\Delta, \gamma^{2 h} I\right)\right)},
$$

where $c_{6}$ is some positive constant. 
Therefore for $h>h_{1}\left(\alpha^{2}\right)$, we have

$$
\{(4.30)\} \leqq \exp \left[c_{7} e^{-c_{6} \bar{B}_{h}^{2}} \gamma^{2 h}|I|\right] \leqq \exp c^{\prime \prime}|I|,
$$

where $c_{7}$ is some positive constant.

Inequality (4.39) together with (4.30) allows us to conclude that for $h>h_{1}\left(\alpha^{2}\right)$ we have

$$
\begin{aligned}
{[I] } & \leqq \exp \left[\left(\tilde{c}+c^{\prime \prime}\right)|I|\right] \exp \hat{V}_{I}^{(h-1)} \\
& \leqq \exp c|I| \cdot \exp \left[\hat{V}_{I}^{(h-1)}+\tilde{C}_{I^{2} \times \mathscr{D}_{h-1}\left(B, \tilde{\varphi}^{(h-1)}\right)}^{(h-1)}\right.
\end{aligned}
$$

which is the thesis of the theorem provided we can drop the condition $h>h_{1}\left(\alpha^{2}\right)$. This condition is in fact unnecessary because $h_{1}\left(\alpha^{2}\right)$ is independent of the cutoff $N$. Therefore when $h \leqq h_{1}\left(\alpha^{2}\right)$, we can rewrite Eq. (4.33) in the following way

$$
\begin{aligned}
\{(4.30)\} & \leqq \prod_{\Delta \subset Q_{h}}\left(1+\sum_{h_{1}}^{\infty} e^{[(4.33)]}+\sum_{h}^{h_{1}} e^{[(4.33)]}\right) \\
& \leqq \prod_{\Delta \subset Q_{h}}\left(1+c \sum_{h}^{h_{1}} e^{[(4.33)]}\right) \leqq \exp \left[c_{8} e^{\left.c 9\left(\lambda_{a \neq f}^{\left(h_{1}\right)}\right)\right)^{4} \gamma^{2\left(h_{1}-h\right)}} \gamma^{2 h}|I|\right],
\end{aligned}
$$

where $c_{9}$ is no longer a negative constant, but in this term $h_{1}$ is fixed, $h \leqq h_{1}$ and there is not any dependence on $N$. Therefore we can conclude again that there exists a constant $c^{\prime \prime \prime}$ such that

$$
\{(4.30)\} \leqq \exp \left[c^{\prime \prime \prime}|I|\right]
$$

This concludes the proof of Theorem 1.

Remarks. a) As was discussed in [1,2] (see Lemma 2 of [1], statement IV) if $\lambda$ is not small enough, we cannot perform the cumulant expansion until $h=0$. In this case there is a certain $\bar{h}\left(N\right.$-independent) below which we just estimate $\hat{V}_{I}^{(\bar{h})}$ in the following way

$$
\hat{V}_{I}^{(\bar{h})} \leqq c_{10}|I|
$$

and then we continue to perform the integration to get rid of the remaining terms $\tilde{C}_{I^{2} \times(\hat{R} \bar{h})^{2}}^{(q)}$ exactly as before.

b) After all the integrations have been performed, we have obtained the upper inequality of the ultraviolet stability, but now there is not any condition on $\alpha^{2}$ except the natural one: $\alpha^{2}<\frac{32}{5} \pi$ which is only due to the fact that we have performed the cumulant expansion until the fourth order, which is needed both for the upper and for the lower bound and can be eliminated by just performing a higher order cumulant expansion and adding the next necessary counterterms.

c) The reader should be aware that although we do not need the positivity of $\bar{W}_{2}^{(h)}\left(\mathscr{D}_{h}\right)$ to control the remaining fourth order terms, this does not imply that this "positivity" property is irrelevant. In fact the positivity of $W_{\mathscr{D}_{h}}^{(2, h)}$ is still fundamental in the proof of inequality (2.1). 


\section{The Observables of the Coulomb Gas in the Regions of Collapse}

The well known connection between the sine-Gordon field model and the twodimensional Coulomb gas is given by the following formal relations

$$
\begin{aligned}
Z_{I}(\varphi) & \equiv \int P(d \varphi) e^{2 \lambda \int: \cos \alpha \varphi(\xi): d \xi} \\
& =\sum_{0}^{\infty} \frac{\lambda^{k}}{k !} \sum_{\varepsilon_{1} \ldots \varepsilon_{k}}^{(-1,+1)} \int_{I^{k}} d \xi_{1} \ldots d \xi_{k}\left\langle: e^{i \alpha \varepsilon_{1} \varphi\left(\xi_{1}\right)}: \ldots: e^{i \alpha \varepsilon_{k} \varphi\left(\xi_{k}\right)}:\right\rangle \\
& =\sum_{0}^{\infty} \frac{\lambda^{k}}{k !} \sum_{\varepsilon_{1} \ldots \varepsilon_{k}}^{(-1,+1)} \int_{I_{k}} d \xi_{1} \ldots d \xi_{k} e^{-\frac{\alpha^{2}}{2}{\substack{\left.\sum_{i, j}, k\right) \\
i \neq j}}_{i \neq\left(\xi_{l}, \xi_{j}\right)}} \\
& =\sum_{0}^{\infty} \sum_{0}^{\infty} \frac{\lambda^{q+p}}{q ! p !} \int_{I^{q}} d x_{1} \ldots d x_{q} \int_{I^{p}} d y_{1} \ldots d y_{p} e^{-\beta U_{(p, q)}\left(x_{1} \ldots x_{q}, y_{1} \ldots y_{p}\right)},
\end{aligned}
$$

where

$$
\begin{aligned}
\langle\cdot\rangle & =\int P(d \varphi), \\
\alpha^{2} & =\beta e^{2},
\end{aligned}
$$

where $\pm e$ is the electric charge of the Coulomb gas particles

$$
\begin{aligned}
U_{(p, q)}\left(x_{1}, \ldots, x_{q} ; y_{1}, \ldots, y_{p}\right)= & -e^{2} \sum_{1}^{q} \sum_{1}^{p} C\left(x_{i}, y_{j}\right) \\
& +\frac{1}{2} e^{2}\left\{\sum_{i \neq l}^{(1, q)} C\left(x_{i}, x_{l}\right)+\sum_{j \neq k}^{(1, p)} C\left(y_{j}, y_{k}\right)\right\},
\end{aligned}
$$

and

$$
C(x, y)=(1-\Delta)^{-1}(x, y),
$$

which at short distances behaves as the two-dimensional Coulomb potential. To make these relations rigorous we have introduced the cutoff field $\varphi^{(N)}$ which amounts to substitution in the last expression of (5.1) of the "Coulomb" twoparticle interaction $C(x, y)$ with the cutoff covariance

$$
C^{(N)}(x, y)=\left[(1-\Delta)^{-1}-\left(\gamma^{2(N+1)}-\Delta\right)^{-1}\right](x, y) .
$$

As

$$
C^{(N)}(0)=\frac{1}{2 \pi} \log \gamma^{N} \equiv \frac{1}{2 \pi} \log l_{N}^{-1},
$$

we can interpret the introduction of this cut-off as the assumption that the particles have a linear size of order $l_{N}=\gamma^{-N}$; of course collapsing phenomena are expected in the limit $l_{N} \rightarrow 0$, that is for $N \rightarrow \infty$.

Let's consider now the term of the grand canonical partition function with $q=p=n$, that is the canonical partition function for the neutral gas with $2 n$ particles, and consider the contribution to $Z_{2 n}^{Q=0}$ from the configurations in which any $+e$ particle is "near" (at a distance of order $l_{N}$ ) to a corresponding $-e$ particle, that is the dipole configurations where each dipole has a momentum of order $e l_{N}$. 
Due to (5.7) the energy of these configurations is approximately

$$
U_{\text {dip }} \simeq-e^{2} \sum_{i}^{n} C^{(N)}(0)=-e^{2} n \frac{1}{2 \pi} \log l_{N}^{-1}=\frac{e^{2} n}{2 \pi} \log l_{N},
$$

and the Gibbs factor is

$$
e^{-\beta U_{d \mu / 2}} \simeq e^{-\frac{\alpha^{2}}{2 \pi} n \log l_{N}}=l_{N}^{-\frac{\alpha^{2}}{2 \pi} n} .
$$

The contribution to the canonical partition function from these configurations is

$$
\begin{aligned}
Z_{2 n}^{(\text {dip })} & \simeq \frac{\lambda^{2 n}}{n !} \int_{I^{n}} d x_{1} \ldots d x_{n}\left(l_{N}^{2}\right)^{n}\left(l_{N}\right)^{-n \frac{\alpha^{2}}{2 \pi}} \\
& =\frac{1}{n !} \lambda^{2 n}\left(l_{N}\right)^{-n \frac{\alpha^{2}}{2 \pi}-2}|I|^{n}=\frac{1}{n !}\left(\lambda \gamma^{N\left(\frac{\alpha^{2}}{4 \pi}-1\right)}\right)^{2 n}|I|^{n},
\end{aligned}
$$

which diverges as $N \rightarrow \infty \quad\left(l_{N} \rightarrow 0\right)$, for $\alpha^{2}>4 \pi$; this means that the dipoleconfigurations give the main contribution to $Z_{2 n}^{Q=0}$ when $l_{N} \ll 1$ if $\alpha^{2} \in[4 \pi, 6 \pi)$. Therefore in this interval the gas looks like a free dipole-gas of activity

$$
\lambda_{\text {dip }}^{(N)}=\left(\lambda \gamma^{\left(\frac{\alpha^{2}}{4 \pi}-1\right) N}\right)^{2}
$$

and with the dipole-momentum of order

$$
e l_{N}=e \gamma^{-N} \text {. }
$$

The density of this dipole-gas is of the order of $\lambda_{\text {dip }}^{(N)}$ which implies that the average distance between two dipoles is

$$
\Delta^{(N)}=\left(\lambda_{\text {dip }}^{(N)}\right)^{-1 / 2}=\lambda^{-1} \gamma^{\left(1-\frac{\alpha^{2}}{4 \pi}\right) N} .
$$

The ratio between the dipole length and the dipoles distance is therefore

$$
O\left(\frac{l_{N}}{\Delta^{(N)}}\right)=O\left(\gamma^{\left(\frac{\alpha^{2}}{4 \pi}-2\right) N}\right) \underset{N \rightarrow \infty}{\longrightarrow} 0, \text { as } \quad \alpha^{2}<8 \pi,
$$

which proves that to consider the dipole-configurations, for $\alpha^{2} \geqq 4 \pi$, as those of a free dipole-gas is consistent.

We can expect similar phenomena when the next even threshold subsequent to $\alpha_{2}^{2}=4 \pi$ are overcome. The next even threshold is $\alpha_{4}^{2}=6 \pi$. Proceeding as before it is easy to realize that when $\alpha^{2}>6 \pi$, an infinite contribution to the partition function comes also from those configurations in which the particles form neutral clusters of four particles so that we can interpret it as the appearance of a free quadrupole gas with the following activity

$$
\lambda_{\text {quad }}^{(N)}=\left(\lambda \gamma^{\left(\frac{\alpha^{2}}{4 \pi}-\frac{3}{2}\right) N}\right)^{4} .
$$

This argument can be repeated each time an even threshold $\alpha_{2 k}^{2}$ is surpassed. It is also easy to realize that contributions from configurations in which particles are assembled in non-neutral clusters never diverge as long as $\alpha^{2}<8 \pi$. The next 
problem is to understand which is the statistical mechanics interpretation of the renormalization procedure; is the renormalized sine-Gordon theory still describing a statistical gas? Which are the natural observables? To get a possible answer let's go back to the sine-Gordon representation for $\alpha^{2}<4 \pi$ and remember that [3], in this case, the density of positive (negative)-charges is given by

$$
\varrho_{ \pm}^{(N)}(x)=\lambda: e^{ \pm i \alpha \varphi^{(N)}(x)}:
$$

that is

$$
\left\langle\varrho_{ \pm}^{(N)}(x)\right\rangle=\lambda \int P\left(d \varphi^{(N)}\right): e^{ \pm i \alpha \varphi^{(N)}(x)}: e^{2 \lambda f: \cos \alpha \varphi^{(N)}(\xi): d \xi} .
$$

Now it is easy to convince ourselves that $\left\langle\varrho_{ \pm}^{(N)}(x)\right\rangle$ diverges as $\alpha^{2} \geqq 4 \pi, N \rightarrow \infty$.

In fact if we consider the generating functional

$$
\int P\left(d \varphi^{(N)}\right) \exp \left(\left[t \lambda \int d x f_{\Delta}(x): e^{i \alpha \varphi^{(N)}(x)}+\lambda \sum_{\varepsilon} \int_{I}: e^{i \alpha \varepsilon \varphi^{(N)}(\xi)}: d \xi\right]\right),
$$

where $f_{\Delta}(x)$ is a function with compact support $\Delta$, it is clear that for $\alpha^{2}>4 \pi$ to prove the ultraviolet stability for (5.18) we have to renormalize the "potential"

$$
\begin{aligned}
\tilde{V}_{0, I}^{(N)} & =t \lambda \int_{I} d x f_{\Delta}(x): e^{i \alpha \varphi^{(N)}(x)}:+2 \lambda \int_{I}: \cos \alpha \varphi^{(N)}(\xi): d \xi \\
& \equiv V_{0, I}^{(N)}+V_{1, \Delta}^{(N)},
\end{aligned}
$$

and if we try to proceed as discussed in [1] we see that now the subtraction constant depends also on $t$ which amounts to a redefinition of the observable

$$
\lambda \int_{I} d x f_{\Delta}(x): e^{i \alpha \varphi^{(N)}(x)}:=\int_{I} d x f_{\Delta}(x) \varrho_{+}^{(N)}(x) .
$$

We have not proven, with the same technique of [1] the ultraviolet stability of (5.18) after the renormalization of the "potential" (5.19), but let's assume that this is only a technical problem and that the results of [1] can be applied also to this case. Let's therefore consider the subtraction constants that would be needed, using the same procedure as in [1]. ${ }^{5}$

For $\alpha^{2}<6 \pi$, we need only one subtraction constant which in this case is:

$$
\left[\frac{1}{2} \mathscr{E}^{T}\left(\tilde{V}_{0, I}^{(N)} ; 2\right)\right]_{t}=\frac{1}{2} \mathscr{E}^{T}\left(V_{0, I}^{(N)} ; 2\right)+\left(\mathscr{E}\left(V_{0, I}^{(N)} V_{1,0}^{(N)}\right)-\mathscr{E}\left(V_{0, I}^{(N)}\right) \mathscr{E}\left(V_{1,0}^{(N)}\right)\right),
$$

and therefore $(5.18)$ becomes

$$
\int P\left(d \varphi^{(N)}\right) e^{t\left(\int d x f \Delta(x): e^{i \alpha \varphi^{(N)}(x):-F(N, \Delta)}\right)} e^{V_{I}^{(N)}},
$$

where

$$
\int_{I} d x f_{\Delta}(x): e^{i \alpha \varphi^{(N)}(x)}:-F(N, \Delta) \equiv \varrho_{+, R}^{(N)}(\Delta)=\int_{I} d x f_{\Delta}(x) \varrho_{+, R}^{(N)}(x)
$$

and

$$
\begin{aligned}
\varrho_{+, R}^{(N)}(x)= & \varrho_{+}^{(N)}(x)-F(N, x)=\lambda: e^{i \alpha \varphi^{(N)}(x)}: \\
& -\left[\mathscr{E}\left(V_{0, I}^{(N),-} V_{1, \Delta}(x)\right)-\mathscr{E}\left(V_{0, I}^{(N),-}\right) \mathscr{E}\left(V_{1,4}(x)\right)\right]
\end{aligned}
$$

5 To be precise, ultraviolet stability allows us to prove $t$-analyticity only for those $\alpha^{1}$ such that the constant counterterms are linear in $t$ 
with obvious notations. Here

$$
\begin{aligned}
F(N, x) & =\lambda^{2} \int_{I} d \xi \sum_{-1}^{N-1} e^{-\alpha^{2} U_{(+,-)}^{(0, k)}(x, \xi)}\left(e^{-\alpha^{2} U_{(+,}^{(k+1,-k+1)}(x, \xi)}\right)_{T} \\
& =\lambda^{2} \int_{I} d \xi\left(e^{-\alpha^{2} U_{(+,}^{(0, N)}(x, \xi)}-1\right) .
\end{aligned}
$$

Let's try now to give a physical interpretation to this renormalized positive charge density $\varrho_{+}^{(N)}{ }_{R}(x)$. Following the previous discussion of the Coulomb gas for $\alpha^{2}$ above $4 \pi$, let's consider for the moment $\alpha^{2} \in[4 \pi, 6 \pi)$, we know that a dipole sea with infinite density (as $N \rightarrow \infty$ ) is formed, therefore we would like to subtract this density from $\varrho_{+}^{(N)}(x)$ to get a finite result in the limit $N \rightarrow \infty$. Let's define the positive charge density due to dipoles at a point $x$ in this way

$$
\begin{aligned}
\varrho_{+}^{\operatorname{dip}(N)}(x) & =\left\{\varrho_{+}^{\operatorname{dep}(n),(N)}(x)\right\}_{n=1}^{\infty}, \\
\varrho_{+}^{\operatorname{dep}(n),(N)}(x) & =e \sum_{\substack{1 \\
p+q}}^{q} \sum_{j}^{p} \delta_{x}\left(x_{i}\right)\left\{\int_{\Delta_{x}} d z \delta_{z}\left(y_{j}\right)-\int_{\Delta_{x}} d z\left\langle\delta_{z}\left(y_{j}\right)\right\rangle_{n}\right\},
\end{aligned}
$$

where $\langle\cdot\rangle_{n}$ is the canonical probability measure of the $n$-particle gas and $\Delta_{x}$ is a volume of linear dimension of order $l_{N}$ centered at the point $x$.

The $\left\langle\varrho_{+}^{\operatorname{dip}(N)}(x)\right\rangle$ should give us the average positive density of charges at the point $x$ due to the dipole sea and therefore we expect that for $\alpha^{2} \in[4 \pi, 6 \pi)\left\langle\varrho_{+}^{\operatorname{dep}(N)}(x)\right\rangle \rightarrow \infty$ for $N \rightarrow \infty$ and that performing the renormalization for the charge density amounts to defining

$$
\varrho_{+, R}^{(N)}=\varrho_{+}^{(N)}(x)-\varrho_{+}^{\operatorname{dip}(N)}(x) .
$$

Going back to the sine-Gordon representation we have, with slightly shortened notations

$$
\left\langle\varrho_{+}^{\operatorname{dip}(N)}(x)\right\rangle=e \int_{\Delta_{x}} d z\left\{\left\langle\sum_{i, j} \delta_{x}\left(x_{i}\right) \delta_{z}\left(y_{j}\right)\right\rangle-\left\langle\sum_{i} \delta_{x}\left(x_{i}\right)\right\rangle\left\langle\sum_{j} \delta_{z}\left(y_{j}\right)\right\rangle\right\},
$$

and

$$
\begin{gathered}
\left\langle\sum_{i, j} \delta_{x}\left(x_{i}\right) \delta_{z}\left(y_{j}\right)\right\rangle=Z_{I}^{-1} \sum_{0}^{\infty} \sum_{0}^{\infty} \frac{\lambda^{p+q}}{p ! q !} p q \\
\cdot \int_{I^{q-1}} d x_{1} \ldots d x_{q-1} \int_{I^{p-1}} d y_{1} \ldots d y_{p-1} e^{-\beta U_{(p, q)}^{(N)}\left(x, \underline{X}_{q-1}: v, \underline{Y}_{p}-1\right)} \\
=\lambda^{2} Z_{I}^{-1} \sum_{0}^{\infty} \sum_{0}^{\infty} \frac{\lambda^{p+q}}{p ! q !} \int_{I^{q}} d x_{1} \ldots d x_{q} \int_{I^{p}} d y_{1} \ldots d y_{p} \int P\left(d \varphi^{(N)}\right) \\
: e^{i \alpha \varphi^{(N)}(x)}:: e^{-i \alpha \varphi^{(N)}(z)}:\left(: e^{i \alpha \varphi^{(N)}\left(x_{1}\right)}: \ldots: e^{i \alpha \varphi^{(N)}\left(x_{q}\right)}:: e^{-i \alpha \varphi^{(N)}\left(y_{1}\right)}: \ldots: e^{-i \alpha \varphi^{(N)}\left(y_{p}\right)}:\right) \\
=Z_{I}^{-1} \int P\left(d \varphi^{(N)}\right)\left(\lambda^{2}: e^{i \alpha \varphi^{(N)}(x)}:: e^{-i \alpha \varphi^{(N)}(z)}:\right) e^{2 \lambda f \cos \alpha \varphi^{(N)}(\xi): d \xi} \\
\left\langle\sum_{i} \delta_{x}\left(x_{i}\right)\right\rangle\left\langle\sum_{j} \delta_{z}\left(y_{j}\right)\right\rangle \\
=Z_{I}^{-2} \int P\left(d \varphi^{(N)}\right) \lambda: e^{i \alpha \varphi^{(N)}(x)}: e^{2 \lambda f_{I}: \cos \alpha \varphi^{(N)}(\xi): d \xi} \\
\quad \int P\left(d \varphi^{(N)}\right) \lambda: e^{-i \alpha \varphi^{(N)}(z)}: e^{2 \lambda f: \cos \alpha \varphi^{(N)}(\xi) d \xi}
\end{gathered}
$$


and therefore at the order $\lambda^{2}$ :

$$
\begin{aligned}
\left\langle\varrho_{+}^{\operatorname{din}(N)}(x)\right\rangle= & \lambda^{2}\left\{\int _ { \Delta _ { x } } d z \left[\int P\left(d \varphi^{(N)}\right): e^{i \alpha \varphi^{(N)}(x)}:: e^{-i \alpha \varphi^{(N)}(z)}:\right.\right. \\
& \left.\left.-\int P\left(d \varphi^{(N)}\right): e^{i \alpha \varphi^{(N)}(x)}: \int P\left(d \varphi^{(N)}\right): e^{-i \alpha \varphi^{(N)}(z)}:\right]\right\} \\
= & \lambda^{2} \int_{\Delta x} d z\left(e^{-\alpha^{2} U_{(+,-)}^{(0, N)}(x, z)}-1\right),
\end{aligned}
$$

which diverges for $\alpha^{2} \geqq 4 \pi$ and whose divergent part coincides with the divergent part of (5.23). Therefore Eq. (5.25) defines an observable which is finite for $\alpha^{2} \in[4 \pi, 6 \pi)$ and describes the charge density for the free charges existing above the dipole-sea. This interpretation can be extended when the second even threshold $\alpha_{4}^{2}=6 \pi$ is overcome. Let's write the order- $t$ contribution of the fourth order counterterm

$$
\left.F(N, x)\left(\lambda^{4}\right)\right|_{t}=\left.\frac{1}{4 !} \mathscr{E}^{T}\left(\tilde{V}_{0, I}^{(N)} ; 4\right)\right|_{t} ^{Q=0}
$$

Now, after simple computations it turns out that the divergent part for this term when $N \rightarrow \infty$ is

$$
\frac{1}{2} \mathscr{E}\left(V_{1,4}^{(N)}(x) V_{0, I}^{(N),-} V_{0, I}^{(N),+} V_{0, I}^{(N),-}\right)-\mathscr{E}\left(V_{1,4}^{(N)}(x) V_{0, I}^{(N)-}\right) \mathscr{E}\left(V_{0, I}^{(N)+} V_{0, I}^{(N)-}\right),
$$

and this term is divergent for $N \rightarrow \infty$ when $\alpha^{2} \geqq 6 \pi$.

Defining now, with obvious notations

$$
\begin{aligned}
\varrho_{+}^{\text {quad }(N)}(x)= & e \sum_{i, j} \delta_{x}\left(x_{i}\right) \int_{\Delta_{x}} d z_{1} \delta_{z_{1}}\left(x_{j}\right) \frac{1}{2} \sum_{l, k} \int_{\Delta_{x}} d z_{2} \delta_{z_{2}}\left(y_{l}\right) \int_{\Delta_{x}} d z_{3} \delta_{z_{3}}\left(y_{k}\right) \\
& -e \sum_{i, l} \delta_{x}\left(x_{i}\right) \int_{\Delta_{x}} d z_{2} \delta_{z_{2}}\left(y_{l}\right)\left\langle\sum_{j, k} \int_{\Delta_{x}} d z_{1} \delta_{z_{1}}\left(x_{i}\right) \int_{\Delta_{x}} d z_{3} \delta_{z_{3}}\left(y_{k}\right)\right\rangle,
\end{aligned}
$$

$\varrho_{+}^{\text {quad }}(N)(x)$ gives the density of positive charges at $x$ due to the presence of the quadrupoles ${ }^{6}$ which are formed for $\alpha^{2} \geqq 6 \pi$ and which tends to infinity for $N \rightarrow \infty$. It is easy to see that at order $\lambda^{4}$

$$
\left\langle Q_{+}^{\text {quad }(N)}(x)\right\rangle=\text { divergent part of }\left.F(N, x)\left(\lambda^{4}\right)\right|_{t} ^{Q=0},
$$

and therefore the subtraction to $\varrho_{+}^{(N)}(x)$ needed for $\alpha^{2} \geqq 6 \pi$ means that

$$
\varrho_{+, R}^{(N)}(x)=\varrho_{+}^{(N)}(x)-\varrho_{+}^{\operatorname{dip}(N)}(x)-\varrho_{+}^{\text {quad }(N)}(x) .
$$

Acknowledgements. I am deeply indebted to G. Gallavotti for his constant advice and his encouragement. I also thank M. Aizenman and G. Benfatto for useful discussions and comments.

6 The definition of $\varrho_{+}^{\text {quad }}(N)(x)$ has some arbitrariness, some other terms (finite in the limit $N \rightarrow \infty$ ) can be added to it 


\section{References}

1. Benfatto, G., Gallavotti, G., Nicolò, F.: On the massive sine-Gordon equation in the first few regions of collapse. Commun. Math. Phys. 83, 387 (1982)

2. Benfatto, G., Cassandro, M., Gallavotti, G., Nicolò, F., Olivieri, E., Presutti, E., Scacciatelli, E.: Ultraviolet stability in Euclidean scalar field theories. Commun. Math. Phys. 71, 95 (1980)

3. Fröhlich, J.: Classical and quantum statistical mechanics in one and two dimensions: twocomponent Yukawa- and Coulomb systems. Commun. Math. Phys. 47, 233 (1976)

4. Korepin, V.E.: The mass spectrum and the $S$ matrix of the massive Thirring model in the repulsive case. Commun. Math. Phys. 76, 165 (1980)

Communicated by K. Osterwalder

Received October 1, 1982 